\title{
Vieses comportamentais em projeções macroeconômicas •
}

\author{
Henry Nasser ${ }^{1}$ \\ Rodrigo De-Losso ${ }^{2}$
}

\section{Resumo}

Este trabalho documenta o otimismo na projeção de PIB e IPCA. Isto é, mostram-se que as projeções de PIB são sistematicamente maiores do que efetivamente acontece, e as projeções de IPCA são sistematicamente menores. Depois testa se há um viés antimanada ligado à hipótese de publicidade para o comportamento dos profissionais que fazem tais projeções. Finalmente, verifica se a revisão das projeções ainda carrega o viés de otimismo identificado anteriormente por meio de uma reação exagerada ou sub-reação no comportamento das revisões. Os resultados confirmam a existência de viés otimista na projeção de PIB e IPCA, e esse viés permanece nas revisões subsequentes para PIB. Porém, a hipótese de publicidade parece não se confirmar nas projeções agregadas por tipos de instituição.

\section{Palavras-Chave}

Vieses. Projeções. PIB. Inflação. Boletim Focus.

\begin{abstract}
This paper documents the optimism in the forecast of GDP and IPCA. That is, it shows that GDP forecasts are systematically higher than what actually happens, and IPCA projections are systematically lower. Then it tests whether there is an anti-herding bias related to a publicity hypothesis regarding institutional incentives for the behavior of professionals who make such forecasts. Finally, it verifies whether the revision of the forecast still carries the previously identified bias. The results confirm the existence of an optimistic bias in the GDP and IPCA projections and this bias remains in the following revisions for GDP. However, the publicity hypothesis seems to be not confirmed for the aggregated forecasts by types of institutions.
\end{abstract}

\section{Keywords}

Biases. Forecast. GDP. Inflation. Boletim Focus.

\section{JEL Classification}

C12. C53. G41.

- Este artigo é oriundo da dissertação de mestrado do primeiro autor apresentada à FGV-EESP, São Paulo, SP - Brasil. Agradecemos os comentários de Bruno C. Giovannetti.

1 Lead Behavior Advisor - CLOO Behavioral Insights Unit - End.: Rua Fradique Coutinho, 212 São Paulo, SP - Brasil - CEP: 05416-000 - E-mail: henrynasser@cloo.pt. - ORCID: https://orcid. org/0000-0001-7245-5184.

2 Professor - Universidade de São Paulo - Faculdade de Economia, Administração e Contabilidade. End.: Avenida Professor Luciano Gualberto, 908 - São Paulo, SP - Brasil - CEP: 05508-010. E-mail: delosso@usp.br - ORCID: https://orcid.org/0000-0002-3461-8621. Recebido: 24/04/2020. Aceito: 28/01/2021. Editor responsável: Rafael Terra de Menezes

(c) (i) (\$) Esta obra está licenciada com uma Licença Creative Commons Atribuição-Não Comercial 4.0 Internacional. 


\section{Introdução}

É de grande importância para empresas, instituições governamentais e indivíduos em geral estabelecer metas e objetivos a serem atingidos dependendo do curso da economia, com impacto na formação de estoques, lançamento ou descontinuidade de produtos, contratações, realização ou não de investimentos, dentre outros. Essas expectativas podem ser ajustadas e melhores cursos de ação executados se os tomadores de decisão soubessem ex-ante o comportamento sistemático enviesado dos agentes econômicos que disponibilizam essas informações para o mercado de maneira pública ou privada e que acabam por influenciar o planejamento estratégico em geral.

Este artigo verifica e quantifica no Brasil o viés de otimismo nas projeções de Índice de Preços ao Consumidor Amplo (IPCA) e Produto Interno Bruto (PIB) a partir de 2000. Para caracterizar os fatores cognitivos que podem levar a um comportamento enviesado, primeiro documenta-se se profissionais do mercado tendem a errar consistentemente a projeção anual de PIB para cima e de inflação para baixo, como ocorre na Europa e Ásia. Em seguida, testam-se se os erros das projeções anuais de PIB e IPCA podem estar relacionados a incentivos desses profissionais de ganhar publicidade (a chamada publicity hypothesis) ao publicarem suas projeções de modo mais diferenciado e extremo ao consenso, ou seja, se existe algum viés antimanada (anti-herding bias) nos erros das projeções de PIB e IPCA a depender da instituição da qual fazem parte. Finalmente, no processo de revisão de suas próprias projeções anuais, testam-se se os profissionais são superconfiantes com revisões positivas ou conservadores com revisões negativas.

Para o estudo, utilizaram-se as séries históricas agregadas das projeções mensais do mercado divulgadas no Boletim Focus, que podem ser encontradas no sítio do Banco Central Brasileiro. Em seguida, cruzaram-se essas informações com um painel contendo projeções desagregadas das instituições por meio de dados mensais compilados e disponíveis nos terminais da Consensus Economics, Bloomberg e Thomson Reuters Eikon.

Seguindo a literatura teórica e testes empíricos realizados, principalmente em estudos na Ásia e Europa, analisou-se, a partir dos dados agregados de expectativas mensais de consenso, a evidência na literatura de que há uma aversão a projeções ruins por parte dos profissionais que fazem parte do consenso oficial. Com isso, quantificou-se e mensurou-se o tamanho do 
erro médio das projeções de consenso, aqui chamado de viés de otimismo, dependendo de quantos meses à frente do dado efetivo anual foram feitas aquelas projeções de PIB e IPCA.

Em seguida, procedeu-se a testes econométricos para as previsões desagregadas por instituição ao longo do tempo, a fim de estudar se bancos de investimento, que figuram entre os principais rankings de desempenho dos times de pesquisa no território nacional, estimam projeções de PIB e IPCA mais extremas e distantes, quando comparadas àquelas projeções advindas de consultorias e firmas de pesquisa independentes, também figurantes nos rankings do Boletim Focus do Banco Central. A ideia é constatar se bancos errariam mais que consultorias, ou seja, se há algum tipo de viés antimanada relacionado à hipótese de publicidade baseada nos incentivos de revelar publicamente suas projeções, a fim de diferenciar seu produto de research e ganhar publicidade. Ressalta-se que as projeções de bancos de investimento utilizadas neste estudo são aquelas que constam em relatórios publicitados aos investidores pelas áreas de Research Sell-Side, e podem diferir das projeções internamente utilizadas por outras áreas dos bancos. Justamente pelo objetivo de alcançar o grande público de investidores, é que podem ser alvo do viés de publicidade.

$\mathrm{Na}$ última parte do trabalho, testa-se, conforme a literatura empírica mostra, se o comportamento das revisões de estimativas ao longo dos meses até a data efetiva de divulgação da taxa de crescimento anual do PIB e do IPCA pode ajudar a explicar se os profissionais são superconfiantes com revisões positivas e/ou conservadores com revisões negativas; ou seja, se há uma assimetria na diferença entre a antiga e a nova projeção dado o sinal da revisão, sendo ela positiva ou negativa, o que estaria em linha com a hipótese trabalhada na literatura de que certo otimismo por parte dos profissionais é refletido não somente no valor absoluto dos vieses de suas projeções, mas também pelo comportamento das suas próprias revisões.

Em resumo, primeiro documenta-se o viés de otimismo na projeção de PIB e IPCA. Segundo, testa-se a hipótese de publicidade para o comportamento dos profissionais que fazem tais projeções. Finalmente, verifica-se se a revisão das projeções ainda carrega o viés otimista identificado anteriormente por meio da reação exagerada ou sub-reação no comportamento das revisões. Os resultados confirmam a existência de viés otimista na projeção de PIB e IPCA e esse viés permanece nas revisões subsequentes de PIB. Porém, a hipótese de publicidade para o viés antimanada parece não se confirmar de modo agregado por tipos de instituição. 


\section{Revisão da literatura}

Não é novidade a existência de erros sistemáticos nas projeções dos profissionais dos principais agregados econômicos ao redor do mundo. Foram realizados estudos sobre essa evidência em diversos contextos e momentos do tempo, com foco tanto em projeções agregadas do consenso, quanto também nas projeções desagregadas para cada uma das instituições participantes destes mercados.

Batchelor (2007) relata, por exemplo, um viés sistemático de otimismo nas projeções de crescimento de PIB e inflação dos profissionais de mercado, chamados de private forecasters, nas economias do G7 entre os anos de 1990-2005, especialmente para Japão, Itália, Alemanha e França. $\mathrm{O}$ artigo mencionado caracteriza este viés de otimismo não só como previsões de PIB real que se provam muito altas e previsões de inflação que se provam muito baixas para uma determinada data, mas que tal comportamento é propagado em diversas séries de previsões ao longo dos anos, mesmo para as projeções agregadas de consenso. Capistrán e López-Moctezuma (2014) relatam comportamento similar para o México entre os anos de 1995-2009. Também Chen et al (2016) mostram, em um estudo realizado para dez economias asiáticas entre os anos de 1995-2012, que os erros das projeções não diferem entre economias avançadas, a exemplo de Singapura, Coreia do Sul e países emergentes como China e Índia.

No contexto brasileiro, Waack (2017) avalia que as projeções de crescimento do PIB também têm sido historicamente otimistas demais e tais erros dependem tanto dos ciclos econômicos como de efeitos de horizonte temporal; sendo relativamente grandes e negativos para os períodos de recessão, e pequenos e pouco significativos para períodos de expansão. Nessa mesma linha, Ager et al (2009) estendem o trabalho de Batchelor (2007) levando em consideração os erros desagregados por instituição e sugerem que o viés otimista significativo encontrado para Itália pode estar relacionado à acentuada desaceleração econômica no período avaliado, algo similar ao que ocorreu no Brasil ao final do período analisado no presente trabalho.

Há pesquisas que levam em conta o arcabouço comportamental, a fim de avaliar se e como as regras de bolso ou estratégias mentais, chamadas de heurísticas, podem influenciar a tomada de decisões dos próprios ana- 
listas e profissionais de mercado, conforme exposto em Amir e Ganzach (1998). Essas heurísticas permitem que indivíduos simplifiquem tarefas complexas de avaliação de probabilidades subjetivas, porém geralmente se desviam das regras estatísticas usuais e objetivas, de acordo com Tversky e Kahneman (1974). Assim, um debate comum nessa linha de estudo se dá sobre a efetividade do desenho da hipótese de expectativas racionais para esses modelos de projeção. Tais comportamentos estariam sendo chamado de "irracionais", conforme visto em DeBondt e Thaler (1990), quando se assume que os analistas deveriam focar na acurácia de suas projeções e que, por consequência, "erros para cima" e "erros para baixo" dever-se-iam anular ao longo do tempo e entre instituições. Em Campbell e Shape (2009) vemos que os profissionais podem "errar racionalmente" se houver outros fatores sendo buscados por eles no processo de revisão, fatores estes que vão além da acurácia. Um exemplo seria a publicidade gerada por previsões extremamente fora do consenso.

De acordo com Amir e Ganzach (1998), tais estudos ao investigarem como estas heurísticas influenciam a previsão das variáveis macroeconômicas (i.e. PIB, inflação, câmbio etc.) e microeconômicas (i. e. resultados de empresas, papéis de dívida corporativa etc.) acabam por ajudar a explicar também a própria dinâmica e comportamento dos mercados financeiros. Todavia, este trabalho não entra na seara de avaliação sobre se o otimismo e/ou conservadorismo dos profissionais são resultado de um comportamento econômico racional ou consequência de uma projeção irracional; parte-se da análise da existência ou não desta evidência e, deste ponto em diante, realizam-se os testes econométricos sobre as projeções e revisões.

Pela ótica comportamental, no tocante aos fatores cognitivos subjacentes a tais previsões, Elliott et al (2008) lançam a discussão sobre se as funções de perda dos profissionais seriam assimétricas, ou seja, se seus comportamentos estratégicos levariam em conta os custos associados a tais erros. Os resultados sugerem que os profissionais estariam sendo avessos a projeções "ruins", entendido como sendo um crescimento de PIB menor que o esperado e uma taxa de inflação acima do projetado, de modo que incorporam essa aversão a perdas em suas previsões. A hipótese dos autores é se a taxa efetiva de crescimento de PIB em determinado ano supera as projeções públicas iniciais de determinado profissional. No final desse período, o custo auferido pelo profissional por um erro de projeção, incluindo perda de credibilidade e valor de imagem 
da instituição por exemplo, será inferior ao do erro se a taxa efetiva for maior do que a projetada inicialmente por ele. A ideia que justifica esse comportamento é que agentes econômicos estariam contentes com uma taxa de crescimento de PIB mais positiva do que esperado. Essa dinâmica também ocorreria para a inflação, porém de maneira oposta: uma taxa de inflação acima do projetado inicialmente seria pior para os agentes de maneira geral, como será explorado na primeira seção do presente trabalho.

Para Campbell e Shape (2009) os erros sistemáticos de previsões de agregados econômicos mensais podem estar relacionados a fatores comportamentais como, por exemplo, a heurística de ancoragem descrita por Tversky e Kahneman (1974). Resulta daí que são enviesadas a partir dos dados efetivos dos meses anteriores, ou seja, os participantes do mercado criam expectativas inconscientemente a partir de um valor inicial após serem expostos a ele, e os ajustes acabam sendo insuficientes para corrigir tais vieses, levando a projeções tendenciosas em relação aos valores iniciais.

Outra linha de estudo agrega o papel dos incentivos dos próprios profissionais ao revelarem publicamente as suas projeções. Broughton \& Lobo (2017) caracterizam como viés antimanada (anti-herding bias) a possibilidade de alguns forecasters tentarem diferenciar suas próprias projeções àquelas de outros times de economia, o que seria evidenciado por projeções publicadas ao mercado (published forecasts) mais distantes de consenso do que as publicadas internamente ou para clientes direcionados (private forecasts). Na mesma linha, Batchelor (2007) descreve esse indício de forma similar a Ehrbeck e Waldmann (1996) como sendo um viés racional (rational bias) ligado à hipótese de publicidade (publicity hypothesis) de certos times de análise econômica, como bancos de investimento por exemplo. Tais equipes poderiam buscar publicar projeções muito distantes do consenso para diferenciar seus produtos de research e chamar a atenção dos outros agentes do mercado. Do mesmo modo, profissionais tenderiam a não revisar frequentemente suas projeções mesmo quando erradas, pois, ao fazê-lo, estariam atestando publicamente que suas projeções estariam constantemente incorretas e, assim, ver-se-ia uma persistência maior dos erros nas projeções ao longo dos meses até a data de divulgação efetiva do dado econômico. No estudo de Laster et al. (1999) também se vê o indício de que alguns forecasters possam mostrar um comportamento estratégico para atrair publicidade. Para os autores, a hipótese de publicidade deve atender a dois requisitos, a saber, primeiro correlação positiva entre recompensa pela publicidade e publicação de previsões extremas; segundo, correlação 
negativa entre recompensa pela publicidade e acurácia das previsões. Tais estudos foram replicados por Jales (2017), Pons-Novell (2003) e Ashiya (2008), que mostraram esse padrão para o comportamento das previsões econômicas de inflação na América Latina, desemprego nos EUA e PIB no Japão, respectivamente. Tal hipótese de publicidade ligada ao viés antimanada será explorada na segunda parte do presente estudo.

Já o terceiro ponto analisado neste trabalho foca sobre como o processo de revisão das projeções pode revelar fatores comportamentais que explicariam um maior ou menor erro das estimativas. Amir e Ganzach (1998) definem os conceitos de reação exagerada (overreaction) e sub-reação (underreaction) como sendo a relação positiva entre a magnitude das revisões e os erros das respectivas projeções no primeiro caso e relação negativa entre as duas variáveis no segundo. Para tanto, mostram que analistas tendem a subestimar revisões negativas e superestimar revisões positivas, ou seja, que o sinal da revisão importa. Quando as revisões são positivas, há uma reação exagerada nas revisões. Já quando as revisões são negativas, há uma sub-reação nas revisões. Tal estudo teve replicações e extensões empíricas feitas para Austrália por Marsden, Veeraraghavan e Ye (2007) e, para o Japão, por Ashiya (2003). No Japão, o autor encontra resultados que apontam para o fato de que os profissionais geralmente revisam suas projeções de modo exagerado quando o sinal da revisão é positivo. $\mathrm{Na}$ Austrália, os autores também encontram razões cognitivas nas heurísticas para suportar a conclusão de que os analistas de mercado tendem a ser muito otimistas, exageram suas previsões quando as revisões são positivas e são conservadores quando as revisões são negativas.

Nas próximas seções será abordado como a estruturação e tratamento dos dados tornou possível tal estudo para o caso brasileiro.

\section{Base de dados}

Três grandes conjuntos de dados foram utilizados no presente trabalho. Primeiramente, as expectativas de mercado agregadas, também chamada de consenso, foram extraídas das médias mensais históricas das projeções anuais de PIB e inflação, IPCA, do Boletim Focus por meio do Sistema Gerenciador de Séries Temporais do Banco Central do Brasil com periodicidade mensal, de 2000 a 2018. Assim, para cada ano, calcula-se a média 
mensal de consenso de 24 meses até 1 mês antes da divulgação efetiva do dado. Presume-se para PIB e IPCA o período 24, sendo o mês de janeiro do ano $t-1$ (ano anterior ao dado efetivo), e o período 1, o mês de dezembro do ano $t$ (mesmo ano do dado efetivo). Essa suposição também é válida para as demais bases descritas a seguir. Assim, como exemplo, temos a expectativa média do consenso para a taxa anual de PIB e IPCA de 2010 desde janeiro de 2009 até dezembro de 2010.

Além disso, a fim de estabelecer um painel amostral abrangente das projeções mensais de PIB real anual e IPCA anual desagregadas por instituição participante do mercado brasileiro, compilaram-se as projeções de cada uma das instituições nacionais e internacionais participantes de três diferentes plataformas: Consensus Forecast, Bloomberg e Thomson Reuters Eikon. Embora as instituições respondentes em cada um dos painéis variem entre as bases, as estruturas são similares: para cada um dos meses de coleta das bases, cada instituição presente no painel projeta o crescimento do PIB e IPCA para o ano corrente e um ano à frente. Portanto, obtém-se a projeção desde 24 meses antes do dado efetivo para cada uma das instituições.

Da Consensus Forecast foram extraídas as projeções mensais desde 2004 até 2018, a partir de um universo amostral incluindo entre 25 a 30 instituições a cada mês, totalizando 40 diferentes instituições durante todo o histórico. Da amostra da Bloomberg foram extraídas as projeções desde 2007 até 2018, de 36 instituições. Da Thomson Reuters Eikon foram incorporadas as projeções de 2012 a 2018, de 60 participantes no total. Foi dado o tratamento em todas as bases, a fim de considerar a projeção de um mês anterior ou posterior, ou média de ambas caso houvesse, caso a projeção não aparecesse em algum dos períodos-chave para a análise do trabalho (isto é 24, 18, 12 e 6 meses anteriores ao dado efetivo).

Foram consideradas no painel de dados não balanceado final somente as instituições que reportaram as expectativas por um período maior que 24 meses, totalizando 81 instituições classificadas posteriormente entre bancos de investimento com forte presença nacional, consultorias e firmas de pesquisa independentes nacionais, outras instituições financeiras nacionais e internacionais, outras firmas de pesquisa independente, agências de risco e seguradoras. Foram feitas comparações das projeções individuais das instituições cujas expectativas possuem sobreposição entre bases e viu-se que há consistência entre as três bases. 
Por fim, as variações anuais efetivas de PIB real e IPCA foram obtidas através do Sistema de Contas Nacionais (SCN) e Sistema Nacional de Índices de Preços ao Consumidor (SNIPC), ambas do Instituto Brasileiro de Geografia e Estatística (IBGE), com periodicidade anual, de 2001 a 2018.

Comparou-se o painel criado e o consenso de mercado do Boletim Focus em termos de projeções absolutas para PIB real e desvio-padrão de projeções, a fim de possibilitar o uso da amostra criada das projeções desagregadas por instituição, como forma de representar o consenso na segunda parte do presente estudo. Nos dois gráficos a seguir, pode-se ver na representação à esquerda a comparação entre o valor da projeção de consenso do Boletim Focus e do painel de instituições criado para os mesmos meses anteriores ao dado de PIB efetivo para cada um dos anos do estudo. $\mathrm{Na}$ imagem à direita é feita a comparação visual da média dos desvios padrões das projeções entre os 24, 18, 12 e 6 meses anteriores para cada um dos anos do dado efetivo de PIB entre o Boletim Focus e o painel elaborado.
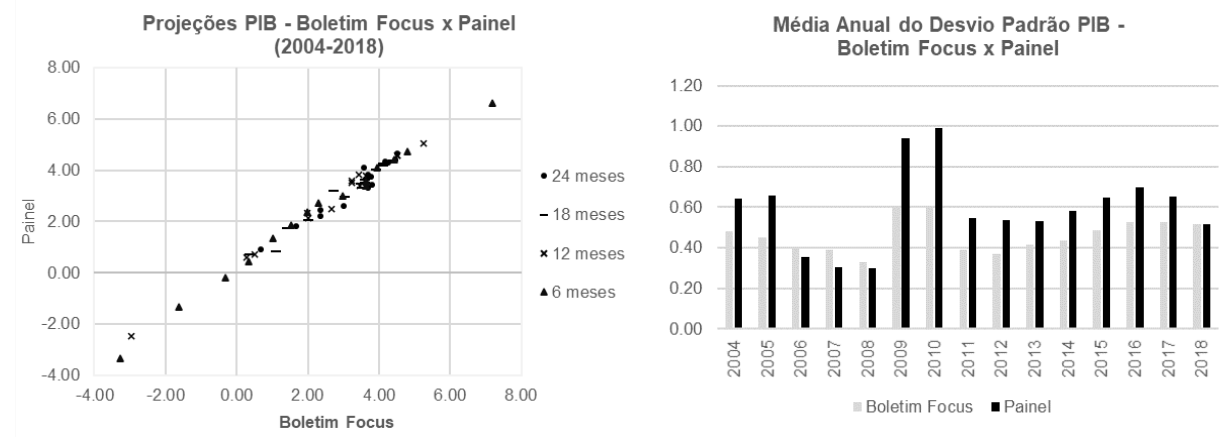

Figura 1 - Comparação Painel x Boletim Focus - Projeção e Desvio Padrão Elaboração a partir do Boletim Focus, Consensus Forecast, Bloomberg, Thomson Reuters Eikon.

Dessa maneira, em termos das médias das projeções, verifica-se que são bastantes similares no período entre 2004-2018, com correlação de 99,3\% entre o painel elaborado e Boletim Focus. Da mesma forma, o desvio padrão médio anual das projeções segue o mesmo desenho entre os anos de 2004-2018, sendo que no painel elaborado o desvio padrão é levemente maior do que o do consenso do Boletim Focus em cerca de 0,13 pontos em média. 


\section{Metodologia}

\subsection{Mensurando o viés de otimismo nas projeções}

Em primeiro lugar, analisaram-se quais anos a curva de projeções para PIB eram negativas, ou seja, apresentavam, de acordo com Batchelor (2007), uma trajetória de walk-down profile. Essa configuração representa um viés otimista dos profissionais em suas primeiras projeções e mostra o ajuste de suas expectativas para baixo com o passar dos meses. De maneira contrária, observou-se em quais anos o desenho das projeções para a inflação era positivo, ou seja, apresentava um walk-up profile dos profissionais, mostrando um viés conservador inicialmente e ajuste de suas expectativas para cima com o passar dos meses até a data efetiva de divulgação.

Assim, a fim de que fosse possível mensurar qual o tamanho do viés de otimismo para os anos em que os profissionais estariam sendo otimistas para PIB e conservadores para inflação, foram feitas regressões com dados em painel, fazendo uso das médias das projeções de consenso agregado 24, 18, 12 e 6 meses antes da divulgação dos dados para as taxas de crescimento de PIB real e inflação para cada ano entre 2001-2018.

Atribuiu-se assim para o PIB o valor de 1 para os períodos de projeções anuais em que os erros da projeção eram positivos, e 0, quando negativos, sendo que o contrário foi feito para os erros de projeção de inflação. Assim, pode-se analisar qual a representatividade dos anos cujas projeções apresentaram um walk-down profile para PIB e walk-up profile para inflação.

Com isto, foram regredidos os erros de previsão por 4 variáveis dummy que representam cada horizonte de previsão e 1 dummy que assume valor de 1 caso naquele ano a tendência das projeções tenha sido positiva para o PIB, e 0 se foi negativa, sendo o contrário para inflação. Formalmente, foi testada a seguinte equação, sendo que, sob a hipótese nula de que não existiria viés, $\alpha_{t}=0$ e tend $=0$ :

$$
\text { EPcons }_{t, T}=\left(\text { Pcons }_{t, T}-y_{T}\right)=\alpha_{t}+\beta_{1} \text { tend }+\varepsilon_{t},
$$

em que

$t=24,18,12$ ou 6.

EPcons é o erro da projeção do consenso $t$ meses antes do dado efetivo em $T$; 
Pcons é a projeção do consenso;

у е́ o dado efetivo daquela variável; e

tend é a dummy que representa se naquele ano as projeções de consenso foram otimistas para PIB e conservadoras para inflação.

\subsection{Viés antimanada ligado à hipótese de publicidade}

Outro fator que poderia ajudar a explicar um maior viés nas projeções é o chamado viés antimanada ligado à hipótese de publicidade. Para tanto, foram utilizadas regressões desagregadas para cada uma das instituições presentes no painel elaborado, em que foram analisadas as projeções para as taxas de crescimento de PIB real e IPCA de cada ano entre 2004-2018, nos 12 meses antes da divulgação do dado.

Criaram-se dummies para cada tipo de instituição, tomando como referência a classificação proposta por Ashiya (2008): (i) bancos de investimento com forte presença nacional "bi", (ii) consultorias e firmas de pesquisa independentes nacionais "fi", (iii) outras instituições financeiras nacionais e internacionais "ob", (iv) outras firmas de research independente "of", (v) agências de risco "ar" e (vi) seguradoras "sg". Maior detalhamento de quais instituições foram incluídas no estudo encontra-se na seção do APÊNDICE A.

A tabela a seguir resume a quebra proposta no trabalho e o número de respondentes que se enquadram em cada tipo de instituição.

Tabela 1 - Número de respondentes por tipo de Instituição

\begin{tabular}{lcc}
\hline Categoria & Siglas & Número de Respondentes \\
\hline Bancos de Investimento & $\mathrm{bi}$ & 19 \\
Firmas de Pesquisa Independente & $\mathrm{fi}$ & 7 \\
Outras Inst. Financeiras e Bancos & ob & 24 \\
Outras Firmas Independentes & of & 23 \\
Agência de Risco & ar & 3 \\
Seguradoras & $\mathrm{sg}$ & 5 \\
\hline Total de Respondestes no Painel & & 81 \\
\hline
\end{tabular}

Elaborado a partir do Consensus Economics, Bloomberg e Thomson Reuters Eikon. 
Desse modo, tomando como base o estudo de Laster et al. (1999) a hipótese de publicidade deve atender a dois requisitos: (1) correlação positiva entre recompensa pela publicidade obtida pela projeção e publicação de previsões extremas; e (2) correlação negativa entre recompensa pela publicidade e acurácia das previsões. Assim, no presente estudo, a hipótese de que haveria a existência de algum viés racional ligado à hipótese de publicidade deve mostrar que (1) o desvio absoluto médio das projeções de consultorias e firmas de pesquisa independentes nacionais será menor que as de bancos de investimento; e (2) apresentam menores erros em suas projeções comparadas bancos de investimento.

Desse modo, foi regredida inicialmente a seguinte equação para os desvios individuais 12 meses antes da divulgação efetiva da taxa de crescimento primeiramente de PIB e depois de IPCA, sendo que, sob a hipótese nula de que não haveria diferença nos desvios frente ao consenso, $\beta_{1}=\beta_{2}$ :

$$
\begin{gathered}
\mid \text { DEVinst }_{12, T}|=| \text { Pinst }_{12, T}-\text { Pcons }_{12, T} \mid=\alpha_{12}+ \\
\left(\beta_{1} \mathrm{fi}+\beta_{2} \mathrm{bi}+\beta_{3} \mathrm{ob}+\beta_{4} \text { of }+\beta_{5} \mathrm{sg}\right)+ \\
\text { Controles Anuais }+\varepsilon_{\text {inst }}
\end{gathered}
$$

Em seguida, regrediu-se a equação a seguir para os erros das projeções individuais 12 meses antes da divulgação efetiva da taxa de crescimento primeiramente de PIB e depois de IPCA, sendo que, sob a hipótese nula de que não haveria diferença nos erros de projeção entre os tipos de instituição $\beta_{1}=\beta_{2}$.

$$
\begin{gathered}
\mid \text { EPinst }_{12, T}|=| \text { Pinst }_{12, T}-\mathrm{y}_{T} \mid=\alpha_{12}+ \\
\left(\beta_{1} \mathrm{fi}+\beta_{2} \mathrm{bi}+\beta_{3} \mathrm{ob}+\beta_{4} \text { of }+\beta_{5} \mathrm{sg}\right)+ \\
\text { Controles Anuais }+\varepsilon_{\text {inst }},
\end{gathered}
$$

em que,

DEVinst é desvio da projeção da instituição frente ao consenso;

EPinst é o erro da projeção da instituição 12 meses antes do dado efetivo;

Pinst é a projeção $T-12$ meses. 


\subsection{Reação exagerada e sub-reação}

Isto posto, a terceira e última parte do presente estudo foca na análise de que o próprio comportamento das revisões individuais de cada instituição apresentaria um fator comportamental importante no que tange aos vieses da projeção a depender do sinal da revisão. Testa-se se há uma reação exagerada da revisão se o coeficiente desta regredido contra o erro da respectiva projeção é positivo, e se há uma sub-reação quando este é negativo.

Para tal análise, foram utilizadas regressões via método dos mínimos quadrados ordinários com dados em painel das projeções desagregadas para cada uma das instituições presentes no painel elaborado, em que foram analisados os erros das projeções para as taxas de crescimento de PIB real e IPCA de cada ano entre 2004-2018, 12 meses antes da divulgação do dado efetivo, e a revisão das mesmas previsões entre os 24 e 12 meses, ambas elevadas ao quadrado dado que a hipótese é de que como a magnitude da revisão se correlacionaria com o erro da projeção 12 meses, dependendo do sinal da revisão.

A seguir, encontra-se a tabela descritiva dos dados analisados seguindo Ashiya (2003):

Tabela 2 - Estatísticas Descritivas de acordo com o Sinal da Revisão

\begin{tabular}{lccc}
\hline PIB & Amostra Total & Revisões Negativas & Revisões Positivas \\
\hline Média das Revisões & -1.01 & -1.83 & 0.84 \\
Desvio Padrão das Revisões & 1.81 & 1.50 & 0.78 \\
\hline Média dos Erros & 0.81 & 1.24 & -0.15 \\
Desvio Padrão dos Erros & 1.76 & 1.66 & 1.60 \\
\hline Total de Observações & 385 & 266 & 119 \\
\hline \multicolumn{4}{c}{} \\
\hline IPCA & Amostra Total & Revisões Negativas & Revisões Positivas \\
\hline Média das Revisões & 0.22 & -0.97 & 0.96 \\
Desvio Padrão das Revisões & 1.36 & 1.09 & 0.92 \\
\hline Média dos Erros & -0.21 & 0.31 & -0.54 \\
Desvio Padrão dos Erros & 1.83 & 1.35 & 2.00 \\
\hline Total de Observações & 348 & 133 & 215 \\
\hline
\end{tabular}

Elaborado a partir de Consensus Economics, Bloomberg e Thomson Reuters Eikon. 
De maneira formal, regrediu-se a seguinte equação, sendo que, sob a hipótese nula, inexiste diferença entre o sinal da revisão para o tamanho do erro, ou seja, testa-se $\beta_{1}=0$ :

$$
\left(\text { EPinst }_{12, T}\right)^{2}=\alpha+\beta_{1}\left(\text { REVinst }_{12, T}\right)^{2}+\varepsilon_{t} \text {, }
$$

em que

$$
\begin{aligned}
& \left(\text { EPinst }_{12, T}\right)^{2}=\left(\text { Pinst }_{12, T}-\mathrm{y}_{T}\right)^{2} \\
& \left(\text { REVinst }_{12, T}\right)^{2}=\left(\text { Pinst }_{24, T}-\text { Pinst }_{12, T}\right)^{2}, \\
& \text { para Rev }>0 \text { e Rev }<0
\end{aligned}
$$

REVinst é a revisão das projeções individuais entre os 24 e 12 meses antes do dado efetivo;

EPinst é o erro de 12 meses associado a cada uma destas revisões.

Inicialmente foi modelada a amostra total contendo revisões individuais positivas e negativas. Em seguida, foi modelada a amostra segregada para as revisões que apresentavam somente sinal negativo, isto é, daqueles profissionais que revisaram suas projeções para baixo. Posteriormente, foi modelada a amostra com aquelas somente com sinal positivo, isto é, daqueles profissionais que revisaram suas projeções para cima.

\section{Resultados}

\subsection{Viés de otimismo nas projeções}

Inicialmente analisou-se para quais anos a curva de projeções de consenso agregado do Boletim Focus para PIB apresentavam um walk-down profile; e, de maneira contrária, para quais anos o desenho das projeções para a inflação apresentava um walk-up profile, representando o viés conservador dos profissionais que inicialmente ajustavam suas expectativas para cima com o passar dos meses. 
Tabela 3 - Vieses na Projeção de Consenso para PIB e IPCA

Dados: 2001-2018

PIB - número de anos cuja projeção ficou:

Acima do dado efeitivo

Abaixo do dado efeitivo

Total

Percentual Histórico - Acima do dado efetivo (\%)

IPCA - número de anos cuja projeção ficou:

Acima do dado efeitivo

Abaixo do dado efeitivo

Total

Percentual Histórico - Abaixo do dado efetivo (\%)

24 meses 18 meses 12 meses 6 meses 3 meses

$\begin{array}{lllll}12 & 13 & 11 & 7 & 8\end{array}$

$\begin{array}{lllll}6 & 5 & 7 & 11 & 10\end{array}$

$\begin{array}{ccccc}18 & 18 & 18 & 18 & 18 \\ 67 \% & 72 \% & 61 \% & 39 \% & 44 \%\end{array}$

24 meses 18 meses 12 meses 6 meses 3 meses

$\begin{array}{lllll}5 & 5 & 7 & 9 & 7\end{array}$

$\begin{array}{lllll}13 & 13 & 11 & 9 & 11\end{array}$

\begin{tabular}{ccccc}
18 & 18 & 18 & 18 & 18 \\
\hline $72 \%$ & $72 \%$ & $61 \%$ & $50 \%$ & $61 \%$
\end{tabular}

Pode-se ver que na maior parte do histórico desde 2001, as projeções de PIB real anuais oficiais do consenso do Boletim Focus apresentam o comportamento walk-down descrito na literatura. Em cerca de 2 a cada 3 anos (mais de 60\% nos 12 meses à frente, por exemplo) as projeções médias de consenso se mostraram acima do real crescimento da economia. De modo contrário, em relação às projeções de IPCA anual, em 2 a cada 3 anos (mais de $60 \%$ também nos 12 meses à frente, por exemplo) as projeções médias de consenso se mostraram abaixo da real taxa de inflação da economia, conforme trajetória também identificada nos gráficos a seguir.

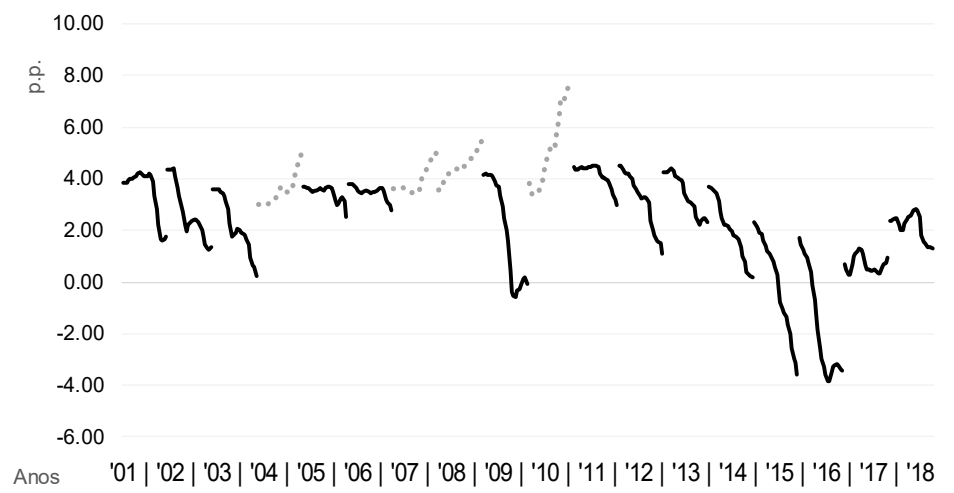

Figura 2 - Trajetória para Projeções de Consenso do PIB real

Note como as projeções vão-se ajustando para baixo em quase todos os anos. 


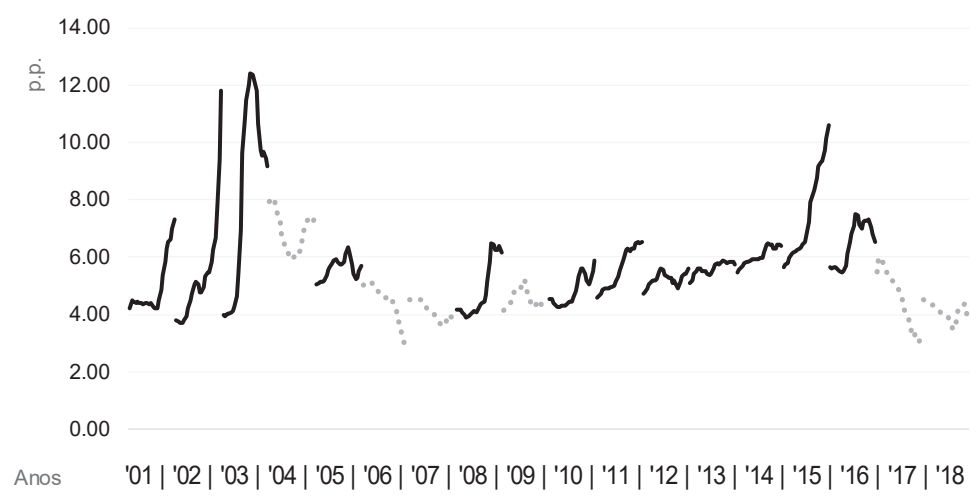

Figura 3 - Trajetória para Projeções de Consenso de IPCA

Note como as projeções de IPCA ajustam-se para cima ao longo do tempo.

Poder-se-ia pensar que tal fato é fruto de uma correlação histórica entre taxas anuais de PIB altas e taxas de inflação baixas, isto é, que ciclos econômicos positivos apresentam historicamente tanto altas taxas de crescimento de PIB quanto baixas taxas de inflação para os mesmos anos. Sabendo disso, profissionais otimistas com os rumos da economia poderiam mirar repetidamente para tal cenário. Porém, os dados mostram uma dissociação entre estas duas variáveis no período recente, conforme representação gráfica a seguir:

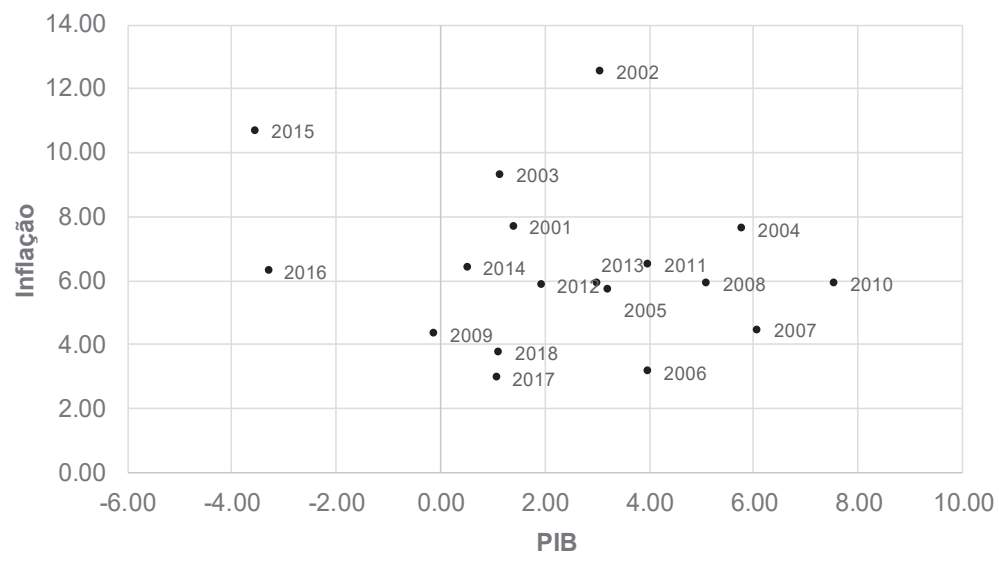

Figura 4 - Relação Anual Histórica entre PIB real e IPCA 
Assim, parte-se para a mensuração de qual o viés médio esperado para cada instante do tempo, tanto para as projeções de PIB real quanto para IPCA. Isto é possível, pois conseguimos identificar a qualquer instante do tempo se o desenho das projeções para aquele ano corrente é negativo ou positivo. Isto significa que se nos encontramos no mês de dezembro de 2019 por exemplo, já sabemos desde janeiro de 2019 se a tendência das projeções para aquele ano ou próximo ano é negativa ou positiva, sendo que no histórico nunca ocorreu uma reversão relevante de tendência entre a projeção de 24 meses antes do dado e sua última previsão.

Tabela 4 - Mensuração do Viés na Projeção do Consenso para PIB

\begin{tabular}{|c|c|c|c|c|c|c|}
\hline Dados: 2001-2018 & Brasil & & & & & \\
\hline Taxa média - PIB & 2.32 & & & & & \\
\hline Desvio-padrão & 2.96 & & & & & \\
\hline Viés na Projeção de Consenso - PIB (p.p.) & viés & p-valor & tend & p-valor & efeito líquido & Wald p-valor \\
\hline 24 meses & $-2.600 *$ & $(0.010)$ & $4.736 * * *$ & $(0.000)$ & 2.14 & 0.0002 \\
\hline 18 meses & $-2.475 * *$ & $(0.007)$ & $4.218 * * *$ & $(0.000)$ & 1.74 & 0.0003 \\
\hline 12 meses & $-1.900 * *$ & $(0.007)$ & $2.850 * * *$ & $(0.001)$ & 0.95 & 0.0009 \\
\hline 6 meses & $-1.075^{*}$ & $(0.020)$ & 1.204* & $(0.021)$ & 0.13 & 0.0208 \\
\hline
\end{tabular}

$* \mathrm{p}<0.05, * * \mathrm{p}<0.01, * * * \mathrm{p}<0.001$

Assim, em média, para os anos em que a projeção de PIB apresenta um viés otimista, a maioria dos casos historicamente no período analisado, a primeira projeção dos profissionais, ou seja, 24 meses antes do dado anual efetivo, está em média superestimando em 2.14 pontos percentuais o real crescimento da economia, e 12 meses antes do dado, a superestimação é da ordem de 0.95 pontos percentuais.

Tabela 5 - Mensuração do Viés na Projeção do Consenso para Inflação

\begin{tabular}{lcccccc}
\hline Dados: 2001-2018 & Brasil & & & \\
\hline Taxa média - IPCA & 6.38 & & & & \\
Desvio-padrão & 2.50 & & & & \\
\hline & & & & & & \\
\hline \\
Viés na Projeção de Consenso - IPCA (p.p.) & viés & p-valor & tend & p-valor efeito líquido & Wald p-valor \\
24 meses & 0.933 & $(0.309)$ & $-3.567^{* *}$ & $(0.005)$ & $\mathbf{- 2 . 6 3}$ & 0.0047 \\
18 meses & 0.767 & $(0.394)$ & $-3.167^{* *}$ & $(0.009)$ & $\mathbf{- 2 . 4 0}$ & 0.0094 \\
12 meses & 0.317 & $(0.737)$ & -1.750 & $(0.143)$ & $\mathbf{- 1 . 4 3}$ & 0.1426 \\
6 meses & $0.0500(0.946)$ & -0.792 & $(0.383)$ & $\mathbf{- 0 . 7 4}$ & 0.3833 \\
\hline
\end{tabular}

$* \mathrm{p}<0.05, * * \mathrm{p}<0.01, * * * \mathrm{p}<0.001$ 
Já para a inflação, a primeira projeção dos profissionais, ou seja, 24 meses antes do dado anual efetivo, está em média subestimando em 2.63 pontos percentuais a taxa de inflação anual da economia; e 12 meses antes do dado, a subestimação é da ordem de 1.43 pontos percentuais, embora estatisticamente insignificante.

\subsection{Viés antimanada ligado à hipótese de publicidade}

A seguir analisa-se se o desvio das projeções contra o consenso das firmas de pesquisa independentes é menor do que o desvio dos bancos de investimento, seguindo a metodologia de Ashyia (2009). A ideia é testar se a publicidade obtida com projeções extremas, descrita na literatura como um dos possíveis fatores a diferenciar as publicações de bancos de investimentos contra as outras instituições de mercado, indica algum viés antimanada ligado à hipótese de publicidade.

Assim, a tabela a seguir indica que o desvio absoluto médio dos bancos de investimento é levemente maior (menos negativo em comparação ao consenso das instituições) do que o desvio das firmas independentes tanto para PIB quanto para IPCA, sendo este o menor entre todas as instituições do painel elaborado. Tal resultado parece indicar que as projeções dos bancos de investimento são levemente mais extremas do que as de firmas independentes, em linha com o indício do estudo de referência, porém tal diferença entre esses dois grupos não é estatisticamente significante. Importante notar que ambos os grupos ficam mais perto do consenso do que as outras instituições que não têm presença nacional relevante ou que possam não atualizar suas estimativas com tanta frequência publicamente. 
Tabela 6 - Desvio versus Consenso na projeção de PIB e IPCA por Instituição

Dados entre 2004-2018

Desvio Médio $12 m$ na Projeção - PIB

Desvio $\quad \mathrm{p}$-valor Wald p-valor

Desvio Médio

$0.798 * * * \quad(0.000)$

Firmas de Pesquisa Independentes

$-0.562 * * * \quad(0.000) \quad 0.242$

Bancos de Investimento

$-0.482 * * *$

Outras Firmas Independentes

$-0.409 * * * \quad(0.001)$

Outras Inst. Financeiras e Bancos

$-0.335 * * \quad(0.006)$

Seguradoras

$-0.335^{*}$

(0.020)

Controles anuais

Sim

$* \mathrm{p}<0.05, * * \mathrm{p}<0.01, * * * \mathrm{p}<0.001$

Dados entre 2004-2018

\begin{tabular}{|c|c|c|c|}
\hline Desvio Médio $12 m$ na Projeção - IPCA & Desvio & p-valor & Wald p-valor \\
\hline Desvio Médio & $0.490 *$ & $(0.013)$ & - \\
\hline Firmas de Pesquisa Independentes & $-0.445^{*}$ & $(0.024)$ & 0.292 \\
\hline Bancos de Investimento & -0.334 & $(0.068)$ & - \\
\hline Outras Firmas Independentes & 0.065 & $(0.728)$ & - \\
\hline Outras Inst. Financeiras e Bancos & -0.170 & $(0.369)$ & - \\
\hline Seguradoras & -0.085 & $(0.714)$ & - \\
\hline Controles anuais & Sim & & \\
\hline
\end{tabular}

$* \mathrm{p}<0.05, * * \mathrm{p}<0.01, * * * \mathrm{p}<0.001$

Equação estimada: $\mid$ DEVinst $_{12, T}|=|$ Pinst $_{12, T}-$ Pcons $_{12, T} \mid=\alpha_{12}+\left(\beta_{1} \mathrm{fi}+\beta_{2} \mathrm{bi}+\beta_{3} \mathrm{ob}+\beta_{4} \mathrm{of}+\beta_{5} \mathrm{sg}\right)+$ Controles Anuais $+\varepsilon_{\text {inst }}$

Além disso, conforme indicado por Laster et al. (1999), a tendência é de que projeções mais extremas levem a erros maiores de projeção. Assim, a tabela a seguir mostra que o erro médio dos bancos de investimento 12 meses antes da divulgação do dado efetivo de PIB e IPCA é levemente maior (menos negativo versus o erro médio) do que o das firmas de pesquisa independentes, sendo estes dois grupos também mais assertivos do que os outros avaliados. 
Tabela 7 - Vieses na projeção de PIB e IPCA por Instituição

Dados entre 2004-2018

Erro Médio $12 m$ vs. Consenso na Projeção - PIB Viés p-valor Wald p-valor Erro Absoluto Médio

Firmas de Pesquisa Independentes $1.837 * * * \quad(0.000)$

Bancos de Investimento

$\begin{array}{lll}-0.600 * * * & (0.000) & 0.727\end{array}$

Outras Firmas Independentes

$-0.570 * * * \quad(0.000)$

$-$

Outras Inst. Financeiras e Bancos

$-0.503 * * * \quad(0.001)$

Seguradoras

$-0.349 *$

(0.022)

Controles anuais

$-0.434^{*}$

(0.016)

Sim

$* \mathrm{p}<0.05, * * \mathrm{p}<0.01, * * * \mathrm{p}<0.001$

Dados entre 2004-2018

Erro Médio $12 m$ vs. Consenso na Projeção-IPCA Viés p-valor Wald p-valor Erro Absoluto Médio $0.632 * * \quad(0.002)$

\begin{tabular}{|lccc|}
\hline Firmas de Pesquisa Independentes & $-0.406^{*}$ & $(0.041)$ & 0.554 \\
Bancos de Investimento & -0.344 & $(0.064)$ & - \\
\hline Outras Firmas Independentes & -0.009 & $(0.958)$ & - \\
Outras Inst. Financeiras e Bancos & -0.273 & $(0.156)$ & - \\
Seguradoras & -0.129 & $(0.582)$ & - \\
Controles anuais & Sim & & \\
\hline
\end{tabular}

$* \mathrm{p}<0.05, * * \mathrm{p}<0.01, * * * \mathrm{p}<0.001$

Equação estimada: $\mid$ EPinst $_{12, T}|=|$ Pinst $_{12, T}-\mathrm{y}_{T} \mid=\alpha_{12}+\left(\beta_{1} \mathrm{fi}+\beta_{2} \mathrm{bi}+\beta_{3} \mathrm{ob}+\beta_{4}\right.$ of $\left.+\beta_{5} \mathrm{sg}\right)+$ Controles Anuais $+\varepsilon_{\text {inst }}$

Assim, pela metodologia proposta por Ashyia (2009) e Laster et al. (1999), embora os resultados possam indicam que os bancos de investimento publicam projeções um pouco mais distantes do consenso do que firmas de pesquisa independentes eles não são discrepantes o suficiente para afirmar tal hipótese da publicidade. Mesmo assim, os erros médios absolutos das projeções 12 meses antes do dado efetivo destes dois grupos de instituição são menores do que para os outros grupos. 


\subsection{Reação exagerada e Sub-reação}

Focando agora no terceiro e último ponto abordado no atual trabalho, a tabela a seguir mostra que o sinal da revisão possui impacto relevante sobre o tamanho do erro médio das projeções. Conforme exposto por Ashyia (2003), é razoável supor que quanto maior a revisão, menor o erro da projeção, pois os profissionais vão ajustando suas expectativas ao longo do processo. Em linha com o autor, o resultado da tabela do PIB parece indicar pouca correlação entre a revisão média e o erro médio na amostra total (dado que o valor é bem próximo de zero, em -0.0861). Porém, contrariamente ao estudo de referência, na presente análise vê-se que ao segregar entre revisões positivas e negativas, os impactos se tornam mais relevantes e apontam para lados opostos.

Tabela 8 - Efeito do tamanho e sinal da revisão sobre erro da projeção

Dados entre 2004-2018

\begin{tabular}{lccccc} 
Erro na Projeção de Consenso - PIB & viés & p-valor & revisão & p-valor & \# revisões \\
Amostra Total & $4.111^{* * *}$ & $(0.000)$ & $-0.0861^{*}$ & $(0.023)$ & 385 \\
Revisões Positivas & $2.230 * * *$ & $(0.000)$ & $0.248^{*}$ & $(0.013)$ & 119 \\
Revisões Negativas & $5.069 * * *$ & $(0.000)$ & $-0.142 * *$ & $(0.002)$ & 266 \\
\hline
\end{tabular}

P-valor entre parênteses $\mid * \mathrm{p}<0.05, * * \mathrm{p}<0.01, * * * \mathrm{p}<0.001$

Dados entre 2004-2018

\begin{tabular}{lccccc} 
Erro na Projeção de Consenso - IPCA & viés & p-valor & revisão & p-valor & \# revisões \\
Amostra Total & $2.966 * * *$ & $(0.000)$ & $0.214 * * *$ & $(0.000)$ & 348 \\
Revisões Positivas & $3.623 * * *$ & $(0.000)$ & $0.372 * * *$ & $(0.000)$ & 215 \\
Revisões Negativas & $1.687 * * *$ & $(0.000)$ & $0.104 *$ & $(0.018)$ & 133 \\
\hline
\end{tabular}

P-valor entre parênteses $\mid * \mathrm{p}<0.05, * * \mathrm{p}<0.01, * * * \mathrm{p}<0.001$

Em linhas gerais, quando as revisões de PIB são negativas, quanto maior a revisão das projeções, menor o erro, indicando certo grau de conservadorismo ou sub-reação por parte dos profissionais. Já quando as revisões são positivas, quanto maior a revisão das projeções, maior o erro, indicando certo grau de superconfiança ou reação em excesso dos profissionais. Já para IPCA, também pode ser visto que o coeficiente aumenta de magnitude 
quando se analisa somente o recorte de revisões positivas, indicando também a reação em excesso, porém para as revisões negativas o coeficiente permanece positivo, embora em menor magnitude, o que não segue a situação de sub-reação encontrada no caso do PIB.

\section{Conclusão}

O presente trabalho procurou aplicar ao contexto brasileiro a análise da evidência da literatura de que existem erros sistemáticos, chamados vieses, nas projeções dos principais balizadores de expectativas macroeconômicas do mercado. Também procurou avaliar até que ponto tais erros de acurácia podem ser resultado da chamada "irracionalidade" dos agentes econômicos ao serem influenciados pelas heurísticas e funções de utilidade assimétricas em relação a perdas. Ademais, procurou avaliar também ser fruto de uma "racionalidade" no comportamento das projeções, conforme diferentes conjuntos de incentivos são apresentados aos profissionais preditores no exercício do processo de professional forecasting.

Em linha com a literatura de referência, procurou-se ao longo do trabalho analisar o comportamento empírico das projeções individuais e de consenso a partir dos dados disponíveis, relacionando com o arcabouço metodológico e ferramentais econométricos, macro e microeconômicos comportamentais nos referidos pontos ao longo do estudo.

Desse modo, na primeira seção foi visto que na maior parte do período analisado após os anos 2000, a trajetória das projeções anuais de consenso foi negativa para PIB e positiva para inflação, o que pode indicar certo nível de otimismo inicial geral médio por parte dos agentes econômicos e certa aversão a projeções ruins por parte dos profissionais, conforme a literatura sugere. Com esses dados em mãos, o tamanho desses vieses foi possível de ser mensurado, o que pode ajudar com que o mercado em geral, incluindo empresas, investidores e governos, possa auferir ex-ante uma ideia da possível trajetória esperada das projeções oficiais do consenso de mercado.

Assim, ao analisar as projeções desagregadas por instituição, foi visto que os grupos têm magnitudes de desvio versus consenso e vieses de projeção diferenciados entre si, porém não foi possível sustentar a hipótese de que 
exista um viés antimanada no que tange à publicidade obtida por bancos de investimento ao publicarem projeções mais extremas em relação ao consenso, tanto para cima quanto para baixo, quando comparados ao outro grupo de firmas de pesquisa independentes.

Isto posto, viu-se também que, de maneira geral, os profissionais se mostram mais confiantes quando suas próprias revisões são positivas e mais conservadores quando suas próprias revisões são negativas ao longo do processo de ajuste de expectativas com o passar dos meses até o dado efetivo. De igual forma ao já exposto na primeira parte do trabalho, tal resultado ajuda a compreender próprio comportamento das revisões de maneira ex-ante, ou seja, lança luz sobre o que esperar das novas projeções e revisões que são publicadas todos os dias, ajudando aos tomadores de decisão se balizarem cada vez melhor nos indicadores econômicos do mercado brasileiro.

O que é curioso nessas constatações é não se observar a correção desses vieses ao longo do tempo. Ou seja, eles parecem-se manter, não obstante os agentes possam perceber que existe um excesso de otimismo nas projeções de PIB e IPCA. O Banco Central precisa corrigir esse viés em suas projeções para determinar a taxa de juros compatível com a meta de inflação.

\section{Possíveis extensões e limitações do trabalho}

De forma geral, o presente estudo procurou se ater às metodologias e ferramentais propostos em cada uma das referências da literatura apontadas nas três seções de testes.

Uma possível extensão a ser elaborada, a fim de corroborar os indícios encontrados na primeira seção do trabalho sobre a aversão a projeções ruins, seria obter não somente as projeções pontuais de cada um dos profissionais em relação ao PIB real e à inflação, mas também o intervalo de probabilidades atribuídas a cada um destes pontos, conforme estudo de Elliott et al. (2008). Tal extensão sobre as funções de utilidades assimétricas ainda não é possível de ser feita com os dados disponíveis para o estudo no contexto brasileiro, mas pesquisas com profissionais de mercado de outros países europeus e nos Estados Unidos como o painel Livingston e a Survey of Professional Forecasters (SPF) já possuem tal informação. 
Além disso, na segunda seção do trabalho sobre os possíveis vieses por instituição, a análise poderá ser desenvolvida e melhor avaliada à medida que a base de dados continue a tomar um corpo mais estruturado, recorrente e com maior regularidade, já que a compilação das projeções nas plataformas encontradas teve início recente a partir de 2004, ganhando forma mais balanceada e acompanhamento mais constante das instituições somente após 2010, em linha com o desenvolvimento do próprio mercado acionário e financeiro nacional.

\section{Referências}

Ager, Philipp, Marcus Kappler e Steffen Osterloh. 2009. "The accuracy and efficiency of the Consensus Forecasts: A further application and extension of the pooled approach." International Journal of Forecasting 25 , no. 1: 167-181. https://doi.org/10.1016/j.ijforecast.2008.11.008

Amir, Eli e Yoav Ganzach. 1998. "Overreaction and underreaction in analysts' forecasts.” Journal of Economic Behavior \& Organization 37, no.3: 333-347. https://doi.org/10.1016/S0167-2681(98)00092-4

Ashiya, Mashiro. 2008. "Strategic bias and professional affiliations of macroeconomic forecasters." Journal of Forecasting 28, no.2: 120-130. https://doi.org/10.1002/for.1095

Ashiya, Mashiro. 2003. "Testing the rationality of Japanese GDP forecasts: the sign of forecast revision matters." Journal of economic behavior \& organization 50, no.2: 263-269. https://doi.org/10.1016/S0167-2681(02)00051-3

Batchelor, Roy. 2007. "Bias in macroeconomic forecasts." International Journal of Forecasting 23, no.2: 189203. https://doi.org/10.1016/j.ijforecast.2007.01.004

Bondt, Werner F. e Richard Thaler. 1990. "Do security analysts overreact?" The American Economic Review 80, no.2: 52-57. Acesso feito em: 09 de Novembro de 2019. https://www.jstor.org/stable/2006542

Broughton, John B., and Bento J. Lobo. 2017. "Herding and anchoring in macroeconomic forecasts: the case of the PMI.” Empirical Economics 55.3: 1337-1355. https://doi.org/10.1007/s00181-017-1306-6

Campbell, Sean D. e Steven A. Sharpe. 2009. "Anchoring bias in consensus forecasts and its effect on market prices." Journal of Financial and Quantitative Analysis 44, no.2: 369-390. https://doi.org/10.1017/ S0022109009090127

Capistrán, Carlos e Gabriel López-Moctezuma. 2017. "Forecast revisions of Mexican inflation and GDP growth." International Journal of Forecasting 30, no.2: 177-191. https://doi.org/10.1016/j.ijforecast.2013.07.016

Chen, Qiwei, Mauro Costantini e Bruno Deschamps. 2016. "How accurate are professional forecasts in Asia? Evidence from ten countries." International Journal of Forecasting 32, no.1: 154-167. https://doi.org/10.1016/j. ijforecast.2015.05.004

Ehrbeck, Tilman e Robert Waldmann. 1996. "Why are professional forecasters biased? Agency versus behavioral explanations.” The Quarterly Journal of Economics 111, no.1: 21-40. https://doi.org/10.2307/2946656

Elliott, Graham, Ivana Komunjer e Allan Timmermann. 2008. "Biases in macroeconomic forecasts: irrationality or asymmetric loss?" Journal of the European Economic Association 6, no.1: 122-157. Acesso feito em: 26 de Agosto de 2019. http://www.jstor.org/stable/40005153.

Jalles, João. 2017. “Inflation forecasts' performance in Latin America.” Review of development finance 7, no.2: 157-168. https://doi.org/10.1016/j.rdf.2017.09.002 
Laster, David, Paul Bennett e In Geoum. 1999. "Rational bias in macroeconomic forecasts.” The Quarterly Journal of Economics 114, no.1: 293-318. Acesso feito em: 07 de Abril de 2019. https://www.jstor.org/stable/2586954

Marsden, Alastair, Madhu Veeraraghavan e Min Ye. 2008. "Heuristics of representativeness, anchoring and adjustment, and leniency: Impact on earnings' forecasts by australian analysts." Quarterly Journal of Finance and Accounting: 83-102. Acesso feito em: 09 de Novembro de 2019. https://www.jstor.org/stable/40473457

Pons-Novell, Jordi. 2003. "Strategic bias, herding behaviour and economic forecasts." Journal of Forecasting 22, no.1: 67-77. https://doi.org/10.1002/for.860

Tversky, Amos e Daniel Kahneman. 1974. "Judgment under uncertainty: Heuristics and biases." Science 185, no.4157: 1124-1131. https://doi.org/10.1126/science.185.4157.1124

Waack, Carlos. 2017. "Viés nos erros das projeções de crescimento real do produto interno bruto do Brasil condicionado pelo estágio do ciclo econômico.” Dissertação de Mestrado, Insper. Acesso feito em: 12 de Agosto de 2019. http://dspace.insper.edu.br/xmlui/handle/11224/2267

\section{APÊNDICE A - Classificação das instituições participantes do mercado}

Conforme exposto no trabalho, tomou-se como referência primordial a classificação proposta por Ashiya (2008), a fim de organizar os grupos de instituições que seriam analisados na seção sobre a possível existência de um viés antimanada. Para isto levou-se em conta os times de pesquisa dos bancos de investimento que tinham presença no território nacional e historicamente figuram nos principais rankings de desempenho de projeções no Brasil, como por exemplo o ranking da revista Institutional Investor, e para as firmas de pesquisa independentes levou-se em conta aquelas que também tinham times com forte presença no território nacional e figuravam nos principais rankings de performance do próprio Boletim Focus do Banco Central.

Outras classificações também foram encontradas na literatura, como em Laster et al. (1999) e Pons-Novell (2003), e ajudaram a balizar a classificação atual adotada, de acordo a finalidade atual do estudo e a disponibilidade dos dados nas plataformas. Abaixo encontram-se as instituições cujas projeções em algum momento do tempo foram consideradas no estudo: 


\begin{tabular}{|c|c|}
\hline Classificação & Instituição \\
\hline Bancos de Investimento & Banco Bradesco \\
\hline Bancos de Investimento & Banco Fator \\
\hline Bancos de Investimento & Banco Safra \\
\hline Bancos de Investimento & Banco Votorantim \\
\hline Bancos de Investimento & Barclays \\
\hline Bancos de Investimento & BofA - Merrill Lynch \\
\hline Bancos de Investimento & Citigroup \\
\hline Bancos de Investimento & Deutsche Bank \\
\hline Bancos de Investimento & HSBC \\
\hline Bancos de Investimento & Itau BBA \\
\hline Bancos de Investimento & JP Morgan \\
\hline Bancos de Investimento & Morgan Stanley \\
\hline Bancos de Investimento & Santander Brazil \\
\hline Bancos de Investimento & BNP Paribas \\
\hline Bancos de Investimento & Credit Suisse Group \\
\hline Bancos de Investimento & UBS \\
\hline Bancos de Investimento & Brasil Plural \\
\hline Bancos de Investimento & Goldman Sachs \\
\hline Bancos de Investimento & Societe Generale \\
\hline Classificação & Instituição \\
\hline Outras Inst. Financeiras & BBVA \\
\hline Outras Inst. Financeiras & Dresdner Kleinwort \\
\hline Outras Inst. Financeiras & Grupo Bursatil Mex \\
\hline Outras Inst. Financeiras & Royal Bank of Scotlan \\
\hline Outras Inst. Financeiras & Standard Chartered \\
\hline Outras Inst. Financeiras & Unibanco \\
\hline Outras Inst. Financeiras & ABN Amro \\
\hline Outras Inst. Financeiras & Commerzbank \\
\hline Outras Inst. Financeiras & Credit Agricole CIB \\
\hline Outras Inst. Financeiras & DekaBank Deutsche \\
\hline Outras Inst. Financeiras & Helaba Bank \\
\hline Outras Inst. Financeiras & MUFG \\
\hline Outras Inst. Financeiras & Scotiabank \\
\hline Outras Inst. Financeiras & Caixa Asset \\
\hline Outras Inst. Financeiras & Desjardins Group \\
\hline Outras Inst. Financeiras & DZBank \\
\hline Outras Inst. Financeiras & Haitong Banco \\
\hline Outras Inst. Financeiras & Handelsbanken \\
\hline Outras Inst. Financeiras & Natl Bk Canada \\
\hline Outras Inst. Financeiras & Nordea \\
\hline Outras Inst. Financeiras & Sicredi \\
\hline Outras Inst. Financeiras & TD \\
\hline Outras Inst. Financeiras & Wells Fargo \\
\hline Outras Inst. Financeiras & Westpac \\
\hline
\end{tabular}

\begin{tabular}{ll} 
Classificação & Instituição \\
Research Independente & 4e Consultoria \\
Research Independente & LCA Consultores \\
Research Independente & M B Associados \\
Research Independente & Macrometrica \\
Research Independente & MCM Consultores \\
Research Independente & Rosenberg Consultoria \\
Research Independente & Tendencias \\
\hline Seguradoras & ING \\
Seguradoras & Nomura Securities \\
Seguradoras & Brasilprev \\
Seguradoras & Mizuho Secs \\
Seguradoras & Sul America \\
\hline Agência de Risco & Moody's Analytics \\
Agência de Risco & Austin Rating \\
Agência de Risco & Fitch Ratings \\
\hline
\end{tabular}

\section{Classificação}

Outras firmas Independentes Outras firmas Independentes Outras firmas Independentes Outras firmas Independentes Outras firmas Independentes Outras firmas Independentes Outras firmas Independentes Outras firmas Independentes Outras firmas Independentes Outras firmas Independentes Outras firmas Independentes Outras firmas Independentes Outras firmas Independentes Outras firmas Independentes Outras firmas Independentes Outras firmas Independentes Outras firmas Independentes Outras firmas Independentes Outras firmas Independentes Outras firmas Independentes Outras firmas Independentes Outras firmas Independentes Outras firmas Independentes

\section{Instituição}

Capital Economics

Datalynk

Eaton

Econ Intelligence Unit

Global Insight

GlobalData

IDEAglobal

IHS Economics

Oxford Economics

Pezco Economics

RGE

Roubini Global Econ

SILCO N/C.R. Contador

Timetric

Action Economics

Continuum Economics

Julius Baer

Pantheon Macro Advisors

Go Assoc

Gradual CCTVM

PwC

Quantitas

Rio Bravo Invest 